\title{
PRINCIPIOS ÉTICOS Y CÓDIGO DE CONDUCTA PARA PERSONAS Y ENTIDADES MEDIADORAS *
}

\author{
Ramón Alzate Sáez de Heredia \\ Universidad del País Vasco \\ Cristina Merino Ortiz \\ Servicio de Mediación Familiar del Gobierno Vasco
}

RESUMEN. En este artículo se propone un código ético a modo de principios inspiradores de la intervención en procesos de gestión de conflictos y, en especial, en mediación. Esta propuesta combina la trayectoria del movimiento internacional en el desarrollo de procesos de calidad en la resolución de conflictos, con cuestionamientos prácticos que genera la intervención directa en la realidad española. Se ofrecen pautas de actuación para desarrollar el buen hacer de la persona mediadora, identificando una serie de situaciones excepcionales en las que, según el contexto y el rol profesional, se cuestionan tales principios y se anima a la adaptación a las necesidades de cada conflicto y a la evolución social.

Palabras clave: código ético, mediación, principios, conducta, límites.

ABSTRACT. This paper proposes an ethical code which aims to provide a series of inspirational principles for those involved in conflict management processes, particularly in the field of mediation. The proposal combines the precepts of the international movement for the development of high-quality conflict resolution processes with more practical questions arising from direct intervention in the Spanish context. Guidelines are provided to help mediators develop their skills, identifying a series of exceptional situations in which, depending on the context and professional role, the principles in play are questioned and mediators are encouraged to adapt to the individual needs of each specific conflict, as well as to social evolution in general.

Keywords: ethical code, mediation, principles, behavior, limits.

* Fecha de recepción: 20 de diciembre de 2009. Fecha de aceptación: 28 de marzo de 2010.

Este trabajo ha sido subvencionado parcialmente por un convenio de colaboración entre el Departamento de Empleo y Asuntos Sociales del Gobierno Vasco y la Universidad del País Vasco/Euskal Herriko Unibertsitatea, dirigido a la elaboración de herramientas de valoración, formación e investigación en el ámbito de la mediación familiar. 
a proliferación de profesionales y entidades que ofrecen una intervención mediadora en nuestro entorno estatal, así como la promulgación de once leyes autonómicas en materia de mediación familiar ${ }^{1}$, nos ha animado a realizar este trabajo desde dos fuentes de interés: la primera y fundamental, nuestra preocupación y compromiso por el desarrollo de los procesos pacíficos de gestión de conflictos tanto desde el plano de la Teoría del Conflicto como desde la intervención directa en procesos de mediación de calidad. La segunda, de gran peso en la condición de docentes, se centra en un interés pragmático en cuanto a ofrecer un documento de referencia ética a todas las personas que en los últimos años se han venido formando en mediación, para que dispongan de una base común que pueda convertirse en generadora de debate y crecimiento profesional, tanto en la actividad individual como colectiva, de este movimiento de Cultura de Paz que engloba la actividad de la mediación.

El presente documento ${ }^{2}$ está basado en el modelo de conducta que defienden tres organizaciones de gran trayectoria internacional en la gestión y resolución de conflictos como son la American Arbitration Association, la American Bar Association y la Association for Conflict Resolution 3 .

En lo que a nuestro entorno geográfico y cultural corresponde, partimos de la premisa de que los procesos de gestión de conflictos son, por definición, flexibles y adaptables a la realidad social y cultural. Por tanto, esta propuesta de código de conducta se plantea desde unos principios generales del buen hacer de la persona mediadora, permitiendo un margen de situaciones excepcionales en las que, atendiendo al contexto situacional y profesional, se puedan cuestionar tales principios y considerando un concepto amplio de la mediación, en su cualidad de proceso de gestión y transformación pacífica de conflictos.

\section{PREÁMBULO}

La mediación se define como un proceso de comunicación que favorece la negociación asistida para tratar, gestionar e incluso resolver una amplia variedad de conflictos,

${ }^{1}$ Leyes autonómicas reguladoras de la Mediación Familiar: Ley 4/2001, de 31 de mayo, de Galicia; Ley 7/2001, de 26 de noviembre, de la Comunidad Autónoma Valenciana; Ley 15/2003, de 8 de abril, de Mediación Familiar de la Comunidad Autónoma de Canarias; Ley 4/2005, de 24 de mayo, del Servicio Social Especializado de Mediación Familiar de la Comunidad Autónoma de Castilla-La Mancha; Ley 1/2006, de 6 de abril, de la Comunidad Autónoma de Castilla y León; Ley 18/2006, de 22 de noviembre, de Mediación Familiar de la Comunidad Autónoma de Illes Balears; Ley 1/2007, de 21 de febrero, de Mediación Familiar de la Comunidad de Madrid; Ley 3/2007, de 23 de marzo, de Mediación Familiar del Principado de Asturias; Ley 1/2008, de 8 de febrero, de Mediación Familiar de la Comunidad Autónoma del País Vasco; Ley 1/2009, de 27 de febrero, reguladora de la Mediación Familiar en la Comunidad Autónoma de Andalucía, y Ley 15/2009, de 22 de julio, de Mediación en el Ámbito del Derecho Privado de Cataluña.

2 El texto que ha inspirado este trabajo fue, en su versión original, elaborado en 1994 por la American Arbitration Association, la sección de Resolución de conflictos de la American Bar Association y la Association for Conflict Resolution. Posteriormente, un comité formado por representantes de las tres organizaciones procedió a la revisión, modificación y aprobación del documento denominado The Model Standards of conduct for mediators en 2005.

3 De manera especial, deseamos agradecer la contribución de la Association for Conflict Resolution (ACR), en concreto a su presidente Michael John Aloi, director ejecutivo Doug Kleine y a los responsables de su sección internacional David Plumb y Luis E. Ore, por su facilitación para la utilización y adaptación del Model Standards of Conflict for Mediators. 
bien sean de relación, de intereses, de información o, incluso, de carácter estructural o de valores. La característica fundamental se centra en promover la autonomía de las partes, en el sentido de que cada persona que participa como protagonista de un proceso de mediación se responsabiliza y decide sobre su situación particular, siendo asistida desde la profesionalidad de una persona o equipo mediador. Precisamente, por este aspecto, la calidad personal y profesional de las personas que ejercen la mediación es de tal trascendencia que requiere la unificación de unos principios y pautas de actuación.

Los principios que a continuación presentamos son de carácter general y serían, por tanto, aplicables a cualquier ámbito de la práctica de la actividad mediadora. Asimismo, tienen tres objetivos:

- Servir de guía a la intervención de la persona mediadora.

- Informar del carácter de este proceso a las personas que eligen la mediación para tratar sus desavenencias.

- Divulgar y promover la mediación como un proceso de gestión de conflictos en el que la sociedad pueda confiar.

La mediación, como proceso de carácter complementario, en ocasiones, o alternativo en otras, a la Administración de Justicia, tiene una finalidad diversa y amplia, en función del modelo o escuela en la que se base. Entre los objetivos genéricos citamos la posibilidad que ofrece a las personas en conflicto de clarificar y describir el propio conflicto, entender las diferentes percepciones y puntos de vista, así como identificar intereses diversos, explorar y generar opciones, acercándose a optar por soluciones, que incluso se conviertan en acuerdos mutuamente satisfactorios para las partes inicialmente enfrentadas en el proceso. Esta visión amplia del concepto de mediación es la que inspira este código. Planteamos una actitud no finalista, en la medida que el proceso sirve para la gestión y transformación, sin que necesariamente se plasme en un acuerdo por escrito que tenga que proceder a una posterior tramitación judicial.

Expuesto el concepto de la mediación en sentido amplio e identificada la diversidad de su ámbito de aplicación, procedemos a desarrollar algunos aspectos previos que faciliten la lectura y comprensión de los principios éticos:

- La secuencia de principios debe ser considerada en su totalidad, sin tener en cuenta el orden de su aparición, es decir, el sentido global de los mismos constituye el código ético o código de conducta.

- Otro aspecto a aclarar es el lenguaje utilizado, especialmente en cuanto a dos conceptos: «deber» y «ser conveniente», en la medida que los matices de tales expresiones puedan modificar su interpretación. Por ello, puntualizamos que cuando se utilice la expresión «deber» hace referencia a una obligación, en el sentido de respetar el buen hacer que este código pretende. Sin embargo, cuando la referencia se trata de «ser conveniente», indica que es altamente deseable, sin llegar a ser obligatorio, dejando al criterio de la persona mediadora y a su experiencia la decisión de su aplicación. En este sentido, recordamos la flexibilidad y necesaria contextualización de este tipo de procesos que requieren una necesaria profesionalización y formación continua. 
- La referencia a la intervención en mediación incluye tanto la actividad profesional individual de la persona mediadora, como el modo de trabajar en comediación, así como la intervención desarrollada por un equipo interdisciplinar de mediación o entidad mediadora.

- Estos principios no hacen mención expresa a la temporalidad de la mediación, es decir, no concreta el número de sesiones límite de un proceso de mediación, ya fueran mínimas o máximas, ni el periodo temporal en el que se deba desarrollar un proceso. Esta omisión es deliberada. El motivo de evitar cualquier regulación temporal, tan cuestionada en foros profesionales, se basa en la diversidad de la regulación legislativa autonómica sobre esta cuestión, existiendo leyes o reglamentos en algunas comunidades autónomas que especifican ambos aspectos, tanto el número de sesiones como el cómputo total de meses en los que se debe finalizar un proceso y su posibilidad de solicitar prórroga. Por ello, en consonancia con el planteamiento ético que recoge este documento, hemos optado por dejar esta cuestión a criterio del buen hacer de la persona mediadora y de la regulación bajo la que actúe, sin perder de vista que el aspecto temporal debe nutrirse de la misma filosofía que la mediación, debiendo ser este aspecto flexible y adaptable tanto al conflicto concreto, como a las personas que lo protagonizan, como al propio espacio o centro en el que tenga lugar el proceso mediador.

- Algunas cuestiones que aparecen en los principios que a continuación se desarrollan han sido también tratadas y reguladas en la legislación europea, estatal, autonómica o en su desarrollo reglamentario, así como en documentos específicos de mediación, tal como es el contrato o compromiso que firman las partes con las personas mediadoras como protocolo de aceptación de los principios y normas del proceso que van a iniciar de forma voluntaria ${ }^{4}$. Esta simultaneidad de fuentes reguladoras de características o principios del proceso prevemos que va a ser frecuente. Sin ánimo de generar ningún conflicto competencial entre leyes, reglamentos, contratos o códigos de conducta, sino manteniendo la pretensión de que este documento facilite y ayude a dilucidar cualquier dilema ético, confiamos en la capacidad de cada persona mediadora de realizar un esfuerzo adaptativo del espíritu de este código a la letra de la ley, preponderando en todo momento la buena fe en su actuación y respetando la voluntad de las partes y su capacidad de autonomía y autocomposición en la gestión del conflicto.

- Por último, este código de conducta y cada uno de sus principios, en la medida que aún no han sido adoptados por un órgano competente (bien de carácter legislativo, Administración Autonómica o Estatal o Colegios Profesionales), no tienen carácter de ley. Sin embargo, el hecho de que estos principios hayan sido propuestos, debatidos y consensuados por organizaciones veteranas en procesos de gestión de conflictos dan luz y orientan la práctica de la mediación, desde una serie de criterios básicos y planteamientos éticos, a tener en cuenta por toda persona que desarrolle su actividad profesional en el campo de la gestión de conflictos y mediación y que desee ofrecerla con calidad.

4 Citamos la regulación de la Unión Europea que ha sido referencia para el desarrollo de la intervención en mediación en España: Recomendación núm. 12/1986, relativa a medidas tendentes a prevenir y reducir la sobrecarga de trabajo de los Tribunales de Justicia; Recomendación (98)1, del Comité de Ministros, a los Estados miembros sobre la Mediación Familiar; Libro verde sobre modalidades alternativas de solución de conflictos en el ámbito del Derecho civil y mercantil; Directiva Europea 2008/52/CE, del Parlamento Europeo y del Consejo, de 21 de mayo de 2008, sobre ciertos aspectos de la mediación en asuntos civiles y mercantiles. 


\section{PRINCIPIOS}

\section{Principio I. Autonomía y autodeterminación de las partes}

I.1. La persona mediadora debe conducir el proceso de mediación basándose en la autonomía de la voluntad de las partes y en su capacidad de autoorganización. Precisamente, la autonomía y capacidad autoorganizativa favorecen la elección de un proceso de gestión de conflictos que requiere de una participación activa, tal como la mediación, en el que las partes llegan de forma voluntaria y libre a una decisión consensuada. En este sentido, las partes pueden ejercer su autodeterminación en cualquier fase del proceso, desde el inicio en la selección de la persona mediadora, durante el diseño del proceso, la participación de otras personas en la mediación, las sesiones individuales o conjuntas, la interrupción del mismo proceso y su finalización con acuerdo escrito o sin él.

I.1.1. Siendo la autonomía de las partes un principio fundamental del proceso de mediación, la persona mediadora debe adaptar tal principio a su deber de conducir y dirigir el proceso con calidad y respetando cada uno de los principios aquí propuestos.

I.1.2. La persona mediadora no puede asegurar que cada parte en el proceso tome sus decisiones basándose en opciones libremente y técnicamente contrastadas. Sin embargo, en la medida que sea posible, es conveniente que la persona mediadora anime a las partes a consultar a entidades o profesionales que tengan un conocimiento técnico sobre cuestiones que se debatan en el proceso.

I.2. La persona mediadora no deberá, bajo ninguna circunstancia, subestimar o dejar de tener en cuenta la opinión de una de las partes, ya sea por razones personales de interés en lograr acuerdos, cuestiones de autoestima profesional en cuanto a logro de acuerdos en mediaciones, aspectos económicos relacionados con tarifas más altas si el proceso se prolonga o se llega a acuerdo, cuestiones de exigencia procedentes de la Administración o entidad que financia o supervisa dichos procesos, la presión de los medios de comunicación o cualquier otra razón que suponga una presión para la persona mediadora o su equipo o servicio.

\section{Principio II. Imparcialidad de la persona mediadora}

II.1. La persona mediadora deberá interrumpir o suspender el proceso en aquellas situaciones en las que no le sea posible conducir la mediación de un modo imparcial. En este sentido, entendemos la imparcialidad como la posibilidad de ejercer la actividad mediadora desde la libertad y ausencia de vínculo con las personas o el tema que se trate. Es decir, la persona mediadora es capaz de manejar las emociones propias y ajenas sin perder su rol por motivos de favoritismos o prejuicios.

II.2. La persona mediadora deberá dirigir el proceso de mediación de un modo imparcial, evitando conductas que trasladen a las partes una apariencia de parcialidad.

II.2.1. La persona mediadora deberá interrumpir el proceso en caso de riesgo de quebrar su imparcialidad por motivos relacionados con las características personales 
de las partes, su pasado, expresión de valores o creencias, modo de interacción en el proceso o cualquier otra razón que le supongan un prejuicio.

II.2.2. La persona mediadora no deberá aceptar regalos, favores, dinero o cualquier otro objeto de valor de una de las partes en el proceso, en la medida que su aceptación ponga en riesgo la percepción de imparcialidad por la otra parte en el conflicto.

II.2.3. La persona mediadora podrá aceptar objetos de regalo, servicios o detalles de una de las partes, que se consideren pertinentes por las normas culturales del contexto social en el que interviene, en la medida que tales prácticas no cuestionen la imparcialidad de la persona mediadora y su percepción por todas las partes que intervienen en el proceso.

II.3. En el caso de que la persona mediadora tenga dificultad de continuar con el proceso de forma imparcial, deberá transmitírselo a las partes e interrumpir el proceso.

\section{Principio III. Conflictos de interés de la persona mediadora con las partes o el asunto a gestionar}

III.1. La persona mediadora deberá evitar un conflicto de interés o la apariencia del mismo, tanto durante el proceso de mediación como posteriormente a finalizarlo. Un conflicto de interés puede presentarse bien por el vínculo con el tema objeto de disputa, o bien por la relación con una parte protagonista del conflicto, ya sea en un momento pasado o actual, en el ámbito personal o profesional, en la medida que sea una cuestión que altere la imparcialidad de la intervención en mediación.

III.2. La persona mediadora deberá formular preguntas razonables y adecuadas dirigidas a obtener información que le permita comprobar si en algún aspecto su intervención pudiera generar un conflicto de interés con las partes o el objeto de mediación. Estas preguntas o cuestionamientos se formularán en función del contexto concreto, en el sentido que se ajusten a la situación específica y a su percepción de riesgo de generar tal conflicto si continúa con la intervención.

III.3. La persona mediadora deberá interrumpir su intervención tan pronto como le sea posible, en la medida que cualquier conflicto real o potencial de interés con las partes o los temas tratados puedan afectar a la imparcialidad del proceso y de su gestión. Tras esta interrupción y aclaración, si las partes están de acuerdo, la persona mediadora puede continuar con el proceso.

III.4. En el mismo sentido que el apartado anterior, si la persona mediadora descubre, una vez iniciada y aceptada la mediación, cualquier situación que pudiera generar un conflicto de intereses deberá finalizar el proceso tan pronto le sea posible. Asimismo, tras esta finalización repentina, acompañada de una aclaración, si las partes están de acuerdo, la persona mediadora puede continuar con el proceso.

III.5. En el supuesto de que un conflicto de intereses pueda ser considerado una amenaza para la imagen de la mediación, entendida ésta como proceso constructivo, imparcial y pacífico de gestión de conflictos, la persona mediadora deberá finalizar el proceso, aun siendo esta finalización contraria al deseo de las partes.

III.6. En este mismo sentido, con el fin de salvaguardar la integridad de la mediación, la persona mediadora no deberá establecer una relación con los participantes 
o los temas a tratar, en la medida que pudieran cuestionar su imparcialidad o ausencia de interés en el asunto. En el caso de que la persona mediadora desarrolle una relación personal o profesional con las partes, así como con otras personas o organizaciones, conectadas con la mediación en la que ha intervenido, será conveniente tener en cuenta factores tales como el tiempo transcurrido una vez finalizado el proceso, el carácter de las relaciones generadas, así como el tipo de servicios ofrecidos.

\section{Principio IV. Competencia, cualificación profesional, habilidades y calidad personal}

IV.1. La persona mediadora deberá intervenir únicamente cuando reúna la cualificación profesional adecuada para atender las necesidades de las partes en conflicto, en la medida que pueda gestionar el conflicto tanto en su aspecto sustantivo como emocional.

IV.1.1. Se podrá seleccionar libremente, a criterio de las partes en conflicto, una persona mediadora del registro o entidad profesional correspondiente, siempre que reúna la competencia y cualificación profesional que permita intervenir con la diligencia y destreza debida. La formación de origen, su especialización y entrenamiento en mediación, las habilidades de comunicación y de gestión de conflictos, su capacidad empática y de adaptación cultural, junto a cualesquiera otra competencia requerida por la tipología de conflictos en los que interviene, así como la formación continua, serán requisitos para ser considerada persona mediadora.

IV.1.2. La persona que anuncie y ofrezca su actividad profesional como mediadora deberá asistir a cursos de formación continua, al menos con una frecuencia bienal, así como participar en grupos profesionales, jornadas, seminarios o talleres que favorezcan la actualización de sus conocimientos con una frecuencia anual.

IV.1.3. Se considera conveniente que la persona que ejerza la actividad profesional de mediación disponga de información sobre cursos de información y formación en procesos de gestión de conflictos, tales como la mediación, así como entrenamiento en habilidades de comunicación y de intervención en situaciones que puedan ser de utilidad para las partes que acuden a mediación.

IV.2. En el caso de que la persona mediadora considerara que no puede seguir interviniendo en el proceso de modo diligente, deberá transmitir a las partes esta cuestión tan pronto como considere adecuado, así como dar los pasos necesarios para continuar el proceso en una sesión posterior o interrumpirlo, temporal o definitivamente.

IV.3. La persona mediadora no podrá intervenir en procesos de este carácter en el supuesto de que se encontrara bajo los efectos de sustancias estupefacientes, alcohol o tratamiento farmacológico que pudiera alterar su capacidad y percepción.

\section{Principio V. Confidencialidad}

V.1. La persona mediadora deberá mantener la confidencialidad de toda la información obtenida en el proceso salvo acuerdo expreso de las partes, en el sentido de que le autoricen conjuntamente a desvelar la información con una finalidad concreta 
e identificada por las partes como deseada. En el caso de que se tratara de procesos multipartes, esta autorización debe ser expresa de cada una de ellas. Otra fuente de excepción a la confidencialidad es por requerimiento legal y en casos expresos.

V.1.1. En el caso de que las partes pacten en el proceso que la persona mediadora puede desvelar alguna información obtenida durante el mismo, se considerará una excepción al principio de confidencialidad.

V.1.2. La persona mediadora no está autorizada a desvelar información sobre el proceso o el modo de evolucionar de las partes, a personas que no participan en la mediación. En este sentido, se informará en caso de ser requerido judicialmente, de si las partes han iniciado el proceso y expresan interés en continuar, así como, en caso de haber finalizado, podrá informar de que se ha alcanzado o no algún acuerdo.

V.1.3. En el supuesto de que la persona mediadora realice actividades relacionadas con la docencia, investigación o evaluación en mediación, deberá proteger el anonimato de las partes y salvaguardar la confidencialidad de aspectos propios de las partes que hayan compartido durante el proceso.

V.2. En aquellos procesos en los que la persona mediadora mantenga sesiones individuales (denominadas caucus en algunos manuales de mediación) con alguna de las partes del conflicto, deberá tratar directamente este asunto de confidencialidad con esta persona con la que mantiene la sesión individual, quedando acordado el pacto de confidencialidad hacia el resto de personas que participan en el proceso o, en caso contrario, la expresa aceptación de que se comparta tal información.

V.3. La persona mediadora deberá facilitar la comprensión entre las partes, así como la importancia de mantener la confidencialidad de lo que se trate en el proceso, con mención expresa, en su caso, de no desvelar los aspectos que se desvelen en el proceso a personas de su entorno próximo, ya sean del ámbito familiar, laboral o social.

V.4. Atendiendo a las circunstancias del proceso, las partes pueden expresar la necesidad de diferentes niveles de confidencialidad ante el proceso. Es decir, las partes pueden pactar sus propias reglas con respecto a la confidencialidad, o por la trayectoria profesional de una persona o entidad mediadora se pueden establecer particularidades en función de personas con las que se pactan (por ejemplo, menores de edad o personas de edad avanzada o en situación de dependencia), así como por circunstancias de protección de menores que estén siendo tratadas en otros centros o instituciones.

\section{Principio VI. Calidad del proceso de gestión de conflictos y mediación}

VI.1. La persona mediadora deberá dirigir el proceso de mediación basándose en estos principios, de modo diligente en la gestión del tiempo, garantizando la seguridad de las partes en su participación, el equilibrio de sus intervenciones, la legitimidad de las partes en aquellos asuntos que vayan a tratar y el respeto mutuo entre todas las personas que intervengan.

VI.2. Es recomendable que la persona mediadora únicamente acepte intervenir en un proceso cuando tenga la formación específica para dirigir y gestionar de modo eficaz los conflictos sobre los que verse su intervención.

VI.3. En este sentido, es conveniente que la persona en su actividad mediadora sólo acepte aquellos procesos que en los que pueda gestionar el conflicto en el contexto 
de la mediación o procesos de gestión de conflictos colaborativos, dejando para otros procesos y a profesionales específicos la intervención centrada en la adjudicación (ya sea arbitraje o defensa en un procedimiento judicial, o en la terapia).

VI.4. La incorporación o cese de la participación de personas en el proceso de mediación será una decisión de las partes protagonistas directas del conflicto, así como de la propia persona o equipo de mediación. Del mismo modo, tanto las partes como la persona mediadora pueden decidir la celebración de una sesión individual con alguna o todas las partes siempre con una función necesaria respecto al proceso general.

VI.5. La persona mediadora promoverá la comunicación clara, eficaz y respetuosa entre las partes y hacia ellas, con especial atención en caso de cualquier circunstancia personal o compartida que pueda alterar el desarrollo del proceso. En este sentido, aspectos relacionados con el conocimiento del idioma o dialecto, cuestiones de capacidad auditiva o de habilidad de comunicación, así como cualquier otra limitación que pueda condicionar la libre decisión o de movimientos de una de las partes en el proceso, debe ser considerada y adaptado, en su caso, el proceso, el lugar o el tiempo de las sesiones con el fin de facilitarles a esas personas el acceso al mismo.

VI.6. El rol profesional que implica la intervención en mediación, así como en otros procesos colaborativos de gestión de conflictos, difiere sustancialmente de otros roles profesionales del ámbito social, psicológico y jurídico. Por ello, la combinación de la actividad mediadora con otras profesiones puede dar lugar a dilemas éticos que deben ser resueltos desde la propia autolimitación de la persona mediadora a intervenir como terapeuta, asesor jurídico o cualquier otra actividad que rompa el principio de imparcialidad hacia las personas que han intervenido en el proceso o que ponga en peligro la confidencialidad del mismo, así como cualquier otro principio intrínseco a estos procesos.

VI.7. Por tanto, la persona mediadora no podrá dirigir o facilitar con esas mismas partes protagonistas otro proceso de gestión de conflictos que no tengan este planteamiento colaborativo y autocompositivo. Es decir, no podrá actuar como representante legal de una de las partes en un procedimiento judicial.

VI.8. Ante un cuestionamiento ético desde la viabilidad de la mediación como proceso adecuado para el conflicto que las partes plantean, la persona mediadora debe informar de la existencia de otros procesos, aun siendo de carácter heterocompositivo o adjudicatario (arbitraje, procedimiento judicial), para que las partes opten libremente con la información necesaria.

VI.9. La persona mediadora no podrá incorporar otra persona del equipo de mediación o colaboradora en el proceso, sin previa consulta y consiguiente aceptación de las partes. Con carácter previo a dicha incorporación, se deberá informar a las partes de dicha decisión o cualquier otro cambio de lugar o tiempo de celebración del proceso, para obtener el consentimiento o negación del mismo.

VI.10. En el caso de que a lo largo del desarrollo de un proceso de mediación se obtuviera la información de alguna de las partes de la intención manifiesta de llevar a cabo un comportamiento constitutivo de ilícito penal o haberlo realizado y esté pendiente de resolución judicial, la persona mediadora valorará la conveniencia de interrumpir el proceso, posponerlo o suspenderlo de forma definitiva.

VI.11. Si se diera la situación de que a lo largo de un proceso de mediación se percibiera la dificultad en la comprensión por una de las partes, incluso limitaciones 
en su participación, la persona mediadora deberá explorar las circunstancias y el potencial de recursos personales que tiene esa persona para considerar la viabilidad de continuar con el proceso, en la medida que esa parte tenga capacidad para comprender la dimensión del proceso y sus efectos, así como su capacidad de participar y tomar de forma autónoma decisiones y alcanzar acuerdos consensuados.

VI.12. Si la persona mediadora tiene conocimiento de que las partes han tenido comportamientos agresivos o episodios de violencia, pudiendo ser éstos de diversa intensidad, deberá valorar y decidir, atendiendo a la situación concreta, la viabilidad de continuar con el proceso, posponerlo o interrumpirlo definitivamente.

VI.13. Si la persona mediadora considera que la conducta o modo de participación de alguna de las partes en el proceso, incluso la propia conducta de la persona mediadora o equipo de mediación, pusieran en peligro el respeto y cumplimiento de estos principios éticos, valorará la viabilidad de continuar con el proceso, pudiéndolo posponerlo o interrumpirlo definitivamente.

\section{Principio VII. Publicidad e imagen de la mediación}

VII.1. La persona mediadora deberá aportar información veraz y clara en todo anuncio que realice sobre su oferta de intervención en procesos de gestión de conflictos, informando de su formación profesional de origen y específica, experiencia en esta área, así como los servicios que ofrece y las tarifas.

VII.1.1. La persona mediadora no podrá incluir ninguna referencia al resultado del proceso o a la expresión de éxito en sus tarjetas de visita, papelería o comunicaciones por internet, en la medida que dichos conceptos pudieran distorsionar la filosofía de base de estos procesos, más centrados en el propio efecto transformativo, que en la percepción de que el resultado signifique necesariamente éxito en la intervención.

VII.1.2. La persona mediadora deberá aportar su formación y cualificación en procesos de gestión de conflictos y mediación a aquellos registros o entidades públicas que regulen la oferta de personas mediadoras en su municipio, provincia o comunidad autónoma, incluso a nivel estatal, en la medida que esta inscripción garantice la calidad de su actividad por estar acreditada y homologada su formación.

VII.2. La persona mediadora no podrá anunciarse como profesional en mediación de modo que pueda confundir su intervención imparcial, en la medida que esté vinculada su actividad a movimientos de reivindicación de determinados derechos a favor de una sola de las partes participantes en el proceso.

VII.3. La persona mediadora no podrá facilitar datos personales de otras personas en la medida que esta información se haya obtenido en el proceso de mediación, excepto en aquellos casos que se haya solicitado autorización expresa para ello.

\section{Principio VIII. Tarifas y cuestiones económicas}

VIII.1. La persona mediadora deberá aportar a cada parte en el proceso la información completa y precisa sobre las tarifas, honorarios y cualquier cuestión económica relacionada con el desarrollo y finalización, en su caso, del proceso. 
VIII.1.1. En el supuesto de que la persona mediadora ofrezca tarifas diferentes en función del tipo de intervención, complejidad o número de personas participantes, tiempo de dedicación o resultado, o cualquier otro factor que determine el diseño del proceso, deberá de anunciarse tal diversidad de honorarios desde el inicio del proceso para que todas las partes tengan conocimiento de ello y puedan establecer el modo en el que se responderá al pago de los mismos.

VIII.1.2. Las tarifas u honorarios de las personas que realizan una actividad privada en mediación deberán constar de forma escrita para el conocimiento de las partes.

VIII.2. La persona mediadora no podrá establecer tarifas o honorarios de un modo que pueda poner en peligro su imparcialidad en el proceso.

VIII.2.1. La persona mediadora no podrá incluir como condición al establecimiento de sus honorarios el resultado al que lleguen las partes en el proceso o la cantidad económica que pacten como acuerdo. Es decir, en ningún momento el carácter del acuerdo de las partes condicionará el precio del proceso, ya que en ese caso se rompería la imparcialidad ante las partes y la neutralidad ante el resultado.

VIII.2.2. Así como se reconoce la posibilidad de que se establezcan diferentes tarifas u honorarios en función de aspectos económicos de carácter individual de las partes (asimilándose al concepto de derecho a justicia gratuita), esta diferencia de honorarios en ningún momento podrá condicionar la diligencia profesional y buen hacer de la persona mediadora en su intervención.

\section{Principio IX. Promoción, divulgación y desarrollo de la mediación}

IX.1. La persona mediadora dirigirá su actuación a promover el desarrollo de la mediación. Con esta finalidad, a continuación se enumeran algunos principios inspiradores de tal desarrollo:

IX.1.1. Favorecer la diversidad profesional, cultural, desde la amplitud de modelos y capacidad de compartir estilos diversos de intervención.

IX.1.2. Promocionar la oferta de mediación para que sea conocida y accesible a todos los agentes sociales que, a su vez, puedan informar y difundir estos procesos a los núcleos de población hacia los que dirigen su intervención.

IX.1.3. Participación en estudios e investigaciones que promuevan el desarrollo de sistemas y modelos que redunden en la mejora de la calidad de estos procesos.

IX.1.4. Participación en foros de debate, jornadas profesionales y docencia, en la medida que permita la reflexión, autocrítica y formación continua.

IX.1.5. Asistir y orientar a nuevas generaciones de profesionales que estén formándose en mediación y requieran de un entrenamiento o tutoría de su aprendizaje.

IX.2. La persona mediadora deberá expresar respeto y colaboración con otros equipos o colectivos mediadores que, aun con diverso enfoque, escuela o modelo teórico de referencia, ofrezcan una intervención profesional ética en procesos de gestión de conflictos. Asimismo, participará en aquellos foros que permitan el intercambio profesional de reflexiones que favorezcan el desarrollo de la mediación y la mejora de calidad en la atención a la población que demanda procesos colaborativos de gestión de conflictos. 


\section{REFLEXIÓN FINAL}

Mediar éticamente significa tomar decisiones basadas en principios y códigos de conducta compartidos por la comunidad de profesionales de la resolución y transformación de conflictos, similares a los propuestos en el presente artículo. Esto requiere valorar cursos de acción alternativos durante el proceso de mediación y decidir cuáles favorecen más los objetivos y valores subyacentes a la mediación. Sin embargo, el criterio para tomar estas decisiones y la elección de lo que es razonable y justo, cuándo y cómo intervenir, la extensión de su intervención, cómo ejercitar las diferentes dimensiones de su poder, quedan en manos de la persona mediadora. Por supuesto que se supone que los modelos de códigos éticos y de conducta como los presentes, sirven para guiar estas decisiones, aunque inevitablemente los modelos están redactados en términos generales y no ofrecen ejemplos específicos ni explicaciones concretas de cómo la persona mediadora debe aplicar las reglas y principios generales en contextos específicos; en último término, es su responsabilidad ética la adecuación de tales principios a su buen hacer. 